\title{
Asistencia y educación en nefrología en épocas de COVID-19: transformando amenazas en oportunidades
}

\author{
Nephrology medical care and education in times of COVID-19: Turning threats into \\ opportunities
}

\author{
Ricardo Silvariño y José Boggia* \\ Centro de Nefrología, Hospital de Clínicas Dr. Manuel Quintela, Universidad de la República, Montevideo, Uruguay
}

Al momento de escribir este editorial, a lo largo del planeta, el número de pacientes infectados por síndrome respiratorio agudo por coronavirus tipo 2 (SARSCoV-2) (confirmados) asciende a 10 millones, con 500,000 fallecidos por enfermedad por coronavirus 2019 (COVID-19) y el 54\% recuperados.

Entre múltiples interrogantes, la única certeza es que después de esta pandemia nada será igual. Los cambios derivados de esta «nueva realidad» afectan y afectarán todas las áreas de la vida de la humanidad, incluyendo la sanitaria, psicológica, económica, educativay asistencial, entre otras.

La resiliencia (del latín resilio, resilire; significa «saltar hacia atrás, rebotar») es una aptitud que adoptan algunos individuos y comunidades que se caracterizan por su postura ante la superación de una adversidad, con el fin de pensar en un mejor futuro. Desde una lectura resiliente, esta «nueva realidad» puede ofrecer oportunidades en nuestra práctica clínica y formación nefrológica en el futuro cercano.

La telemedicina es definida por la Organización Mundial de la Salud como «la prestación de servicios de atención médica, donde la distancia es un factor crítico, por parte de los profesionales de toda la atención médica que utilizan tecnologías de la información y comunicación para el intercambio de información válida para el diagnóstico, tratamiento y prevención de enfermedades y lesiones, investigación y evaluación, y para la educación continua de los proveedores de atención médica, en aras de promover la salud de las personas y sus comunidades»" Esta definición surge, en parte, del documento elaborado en 1996 en EE.UU. por el Institute of Medicine (IOM) (The National Academies of Sciences Engieneering Medicine). Este, después de revisar los conceptos desarrollados a esa fecha, definió la telemedicina como «el uso de tecnologías electrónicas de información y comunicación para proporcionar y apoyar la atención médica en las situaciones en las que la distancia separa a los participantes»?2.

Múltiples experiencias de telemedicina en nefrología se han implementado en las últimas dos décadas. Entre las más relevantes destacan aquellas dirigidas al control clínico a distancia de pacientes con enfermedad renal crónica $^{3-5}$, interconsulta al nefrólogo desde el primer nivel de atención ${ }^{6,7}$, control periódico de pacientes incluidos en programas de diálisis peritoneal ${ }^{8-12}$, manejo protocolizado de complicaciones de la diálisis peritoneal ${ }^{13}$, control de hemodiálisis (HD) domiciliaria y reclutamiento de pacientes para las listas de trasplante renal $^{5}$, entre otras. Además de estas estrategias asistenciales directas, se han desarrollado programas dirigidos a potenciar la asistencia nefrológica

\footnotetext{
Correspondencia:

Fecha de recepción: 07-07-2020

*José Boggia

Fecha de aceptación: 29-07-2020

Disponible en internet: 17-12-2020

DOI: 10.24875/NEFRO.M20000008

Nefro Latinoam. 2020;17:52-54

E-mail: ppboggia@gmail.com

www.nefrologialatinoamericana.com

2444-9032/C 2020 Sociedad Latinoamericana de Nefrología e Hipertensión. Publicado por Permanyer. Este es un artículo open access bajo la licencia CC BY-NC-ND (http://creativecommons.org/licenses/by-nc-nd/4.0/).
} 
interdisciplinaria ${ }^{14,15}$, educar a grupos de pacientes en el conocimiento y manejo de opciones de terapia de reemplazo renal (TRR) y optimizar las estrategias de autocuidado de pacientes en TRR ${ }^{16,17}$. Algunas de estas estrategias se ven auxiliadas por dispositivos de telemedicina que permiten monitorizar a distancia y en forma automática parámetros objetivos, como peso, presión arterial, temperatura, saturación de oxígeno e, incluso, la diálisis domiciliaria y el diagnóstico mediante telepatología ${ }^{5}$. La mayor parte de estas iniciativas tuvieron un impacto positivo en los destinatarios ${ }^{18,19}$, mostraron una performance equivalente o superior a la consulta presencial en el control nefrológico ${ }^{5}$ y se mostraron como costo-efectivas en términos de economía de la salud ${ }^{5,20,21 .}$

En lo que se refiere a la educación médica de grado y posgrado, la educación a distancia permitió evitar la interrupción completa de cursos y la cancelación de congresos en el contexto de la pandemia por COVID-19. La casi totalidad de las herramientas tecnológicas se han empleado de uno $u$ otro modo con este fin. Estas incluyen herramientas de comunicación semiprivadas (WhatsApp [Mountain View, CA], Slack [Canadá], Viber [Israel], Google Hangouts [Mountain View, CA] $)^{22}$, software para video conferencias ${ }^{23}$, plataformas social-media (Facebook, Twitter) ${ }^{24}$ y herramientas de educación médica de libre acceso (webinarios, etc. $)^{23,25,26}$. Aquellas universidades que no habían desarrollado aún ofertas de educación a distancia debieron generarlas y las que ya tenían plataformas de educación a distancia (p. ej., basadas en Moodle) debieron potenciarlas para ofrecer cursos $100 \%$ virtuales. Esto exigió significativamente las capacidades de las herramientas de software y hardware disponible. Contrastando con la estancada realidad del confinamiento de otros ciudadanos y otras profesiones, los médicos vimos incrementada la demanda de trabajo, que nos exigió la asistencia y el desarrollo urgente y el aprendizaje inmediato de protocolos de actuación para identificar casos sospechosos y minimizar el contagio de los casos confirmados. Esto incluye el aprendizaje de las herramientas y técnicas de telemedicina. Pero a esta vertiginosa exigencia de adaptación laboral asistencial se sumó, en los profesionales universitarios, el desarrollo y aprendizaje de estrategias educativas a distancia. Por otra parte, muchas sociedades científicas cancelaron sus congresos y otras realizaron ofertas de congresos virtuales o potenciaron cursos virtuales. Son ejemplos los cursos virtuales de Sociedad Latinoamericana de Nefrología e Hipertensión (SLANH), el congreso Asociación Renal Europea-Asociación
Europea de Trasplante y Diálisis (ERA-EDTA), la reunión anual de la Glosen, entre otros. Merece destacarse que la mayor parte de estas experiencias han sido evaluadas positivamente por docentes, estudiantes, instituciones y organizadores. Aunque no existen evaluaciones formales del impacto económico, tenemos la certeza que la educación a distancia impacta positivamente. El ahorro de tiempo para los estudiantes surge del ahorro de tiempo de desplazamiento y de búsqueda de información, y del abaratamiento de los cursos, ya que pueden prescindir de infraestructura edilicia, que se sustituye por las plataformas virtuales. Para los docentes, instituciones y organizaciones implica una inversión mayor de tiempo y dinero inicial, pero que se amortiza en el mediano plazo, además del ahorro en costos de infraestructura y personal accesorio, como de limpieza y vigilancia. Además, después de la inversión inicial para el desarrollo de las plataformas educativas y la creación de cursos virtuales, el mantenimiento y renovación de cursos requiere menos inversión.

La «nueva realidad» ha valorizado significativamente la asistencia ${ }^{1}$ y la adquisición del conocimiento en «la virtualidad ${ }^{26}$. Ha obligado a distintos sistemas de salud a poner a punto muchas de las herramientas mencionadas. Ha determinado el desarrollo de un «nuevo lenguaje» de comunicación con los pacientes, estudiantes y entre los integrantes de la comunidad nefrológica ${ }^{24}$. Ha impulsado la creación de decretos y leyes de telemedicina en algunos países de la región (p. ej., Colombia y Uruguay). Ha catalizado la transformación de la educación universitaria en muchos países de Latinoamérica desde sistemas totalmente presenciales hacia propuestas educativas semipresenciales o $100 \%$ virtuales apoyadas por espacios virtuales de aprendizaje.

¿Qué desafíos tiene en esta línea la comunidad nefrológica latinoamericana?

Considerando los aspectos positivos para la comodidad de los pacientes, la mayor frecuencia en el control de la enfermedad crónica y la maximización de los tiempos dedicados por los recursos humanos en salud resaltados antes, podemos prever que una proporción de estos cambios llegaron para permanecer en el largo plazo. Es probable que las consultas presenciales con los pacientes y las instancias educativas presenciales con los estudiantes de grado, así como los desplazamientos para asistir a congresos y cursos de actualización se valoricen, reservando estas instancias para cuando la presencialidad aporte en forma significativa por sobre lo virtual. Los próximos años estarán 
signados por la formalización del aprendizaje de la asistencia y educación virtual, así como su legislación.

Finalmente, debemos destacar que escribimos este editorial desde un país donde la infección por SARSCoV-2 ha determinado pocos casos y menos fallecidos. Así, es probable que el impacto sanitario incluya aún mayores exigencias en otros países de América Latina.

\section{Bibliografía}

1. Rohatgi R, Ross MJ, Majoni SW. Telenephrology: current perspectives and future directions. Kidney Int. 2017;92(6):1328-33.

2. Telemedicine. Washington, D.C.: National Academies Press, 1996.

3. Palen TE, Price D, Shetterly S, Wallace KB. Comparing virtual consults to traditional consults using an electronic health record: An observational case-control study. BMC Med Inform Decis Mak. 2012;12(1):1.

4. Narva AS, Romancito G, Faber T, Steele ME, Kempner KM, Managing CKD by Telemedicine: The Zuni Telenephrology Clinic. Adv Chronic Kidney Dis. 2017;24(1): 6-11.

5. Gudsoorkar P. Telemedicine \& Nephrology. [Online]. 2020. Disponible en: https://www.renalfellow.org/2020/01/24/telemedicine-nephrology/.

6. Zuniga C, Riquelme C, Muller H, Vergara G, Astorga C, Espinoza M. Using Telenephrology to Improve Access to Nephrologist and Global Kidney Management of CKD Primary Care Patients. Kidney Int Reports. 2020;5(6):920-3.

7. Horner K, Wagner E, Tufano J. Electronic consultations between primary and specialty care clinicians: early insights. Issue Brief (Commonw Fund). 2011;23:1-14

8. Li L, Perl J. Can Remote Patient Management Improve Outcomes in Peritoneal Dialysis? Contrib Nephrol. 2019;197:113-23.

9. Milan Manani S. Rosner MH, Virzì GM, Giuliani A, Berti S, Crepaldi C et al. Longitudinal Experience with Remote Monitoring for Automated Peritoneal Dialysis Patients. Nephron. 2019;142(1):1-9.

10. Bunch A, Vesga JI, Camargo DO, Corzo L, Molano AP, Devia ME, et al. Remote Automated Peritoneal Dialysis Management in Colombia. Kidney Int Reports. 2019;4(6):873-6.
11. Wallace EL, Rosner $M H$, Alscher MD, Schmitt $C P$, Jain A, Tentoriet $F$, et al. Remote Patient Management for Home Dialysis Patients. Kidney Int Reports. 2017;2(6):1009-17.

12. Agarwal S, Wilkie M. Remote Patient Management in Peritoneal Dialysis: Opportunities and Challenges. Contrib Nephrol. 2019;197:54-64.

13. Paniagua R, Rojas M, Ramos A. Managing Peritoneal Dialysis Complications through Remote Patient Management Protocols. Contrib Nephrol. 2019;197:65-72.

14. Ishani A, Christopher J, Palmer D, Otterness S, Clothier B, Nugent S, et al. Telehealth by an Interprofessional Team in Patients with CKD: A Randomized Controlled Trial. Am J Kidney Dis. 2016;68(1):41-9.

15. Gordon EJ, Fink JC, Fischer MJ. Telenephrology: A novel approach to improve coordinated and collaborative care for chronic kidney disease. Nephrol Dial Transplant. 2013;28(4):972-81.

16. Malkina A, Tuot DS. Role of telehealth in renal replacement therapy education. Semin Dial. 2018;31(2):129-34.

17. Rosner $\mathrm{MH}$, Lew $\mathrm{SQ}$, Conway $\mathrm{P}$, Ehrlich J, Jarrin $\mathrm{R}$, Patel UD, et al. Perspectives from the kidney health initiative on advancing technologies to facilitate remote monitoring of patient self-care in RRT. Clin J Am Soc Nephrol. 2017;12(11):1900-09.

18. Liddy C, Afkham A, Drosinis P, Joschko J, Keely E. Impact of and satisfaction with a new eConsult service: A mixed methods study of primary care providers. J Am Board Fam Med. 21015;28(3):394-403.

19. Magnus M, Sikka N, Cherian T, Lew SQ. Satisfaction and improvements in peritoneal dialysis outcomes associated with telehealth. Appl Clin Inform. 2017;8(1)214-25.

20. Rosner MH, Khan S. Health Economic Implications of Remote Patient Management. Contrib Nephrol. 2019;197:133-42.

21. Lew SQ, Sikka N, Thompson C, Magnus M. Impact of remote biometric monitoring on cost and hospitalization outcomes in peritoneal dialysis. $J$ Telemed Telecare. 2019;25(10):581-6.

22. Pandya A, Elrggal ME, Jhaveri KD. Use of Semiprivate Smartphone Communication Applications in Nephrology Education. Semin Nephrol. 2020;40,(3);303-8.

23. Farouk SS. Medical Education During the COVID-19 Pandemic: Learning from a distance. Adv Chronic Kidney Dis. 2020 Jun 23.

24. Diniz H, Melilli E. The rise of \#SocialMedia in the Nephrology world. Nefrología. 2020.

25. Jain G, Ahmad M, Wallace EL. Technology, telehealth and nephrology: The time is now. Kidney. 2020 Jun 3.

26. Colbert GB, Topf J, Jhaveri KD, Oates T, Rheault MN, Shah S, et al., The Social Media Revolution in Nephrology Education. Kidney Int Reports. 2018;3(3):519-29. 